\title{
Selection inversion: a probable tool against antibiotic resistance
}

This article was published in the following Dove Press journal: Infection and Drug Resistance

\author{
Zulqarnain Baloch ${ }^{1, *}$ \\ Bilal Aslam 2,* \\ Saima Muzammil ${ }^{2}$ \\ Mohsin Khurshid ${ }^{3}$ \\ Muhammad Hidayat Rasool ${ }^{2}$ \\ $\mathrm{Ke} \mathrm{Ma}^{4}$ \\ 'College of Veterinary Medicine, \\ South China Agricultural University, \\ Guangzhou, China; ${ }^{2}$ Department of \\ Microbiology, Government College \\ University Faisalabad, Faisalabad, \\ Pakistan; ${ }^{3}$ College of Allied Health \\ Professionals, Directorate of Medical \\ Sciences, Government College \\ University Faisalabad, Faisalabad, \\ Pakistan; ${ }^{4}$ College of Traditional \\ Chinese Medicine, Shandong \\ University of Traditional Chinese \\ Medicine, Jinan, China \\ *These authors contributed equally to \\ this work
}

Correspondence: Bilal Aslam

Department of Microbiology,

Government College University

Faisalabad, Faisalabad, Pakistan

Tel +92300747 I09|

Email drbilalaslam@gcuf.edu.pk

$\mathrm{Ke} \mathrm{Ma}$

College of Traditional Chinese, Medicine, Shandong University of Traditional

Chinese Medicine, 4655 University Road,

Changqing, Jinan, Shandong 250355,

China

Tel +86 53I 89628077

$\mathrm{Fax}+8653189628077$

Email make-198807I0@I63.com

\begin{abstract}
Antibiotic therapy has a dual impact: wanted, in which it immediately inhibits the growth of bacteria and the unwanted, which is responsible for the evolution of antibiotic resistance. The dissociation of therapeutic effectiveness from the possible risk of the antibiotic resistance may be attained by taking the advantage of specific relations between these drugs, and the methods in which mutations associated with resistance against a specific antibiotic may modify these relations or it may increase the sensitivity of the bacterium to the other antibiotics. Although the practical implementation of this notion needs considerable advancement and confirmation that depends upon the improvements in the field of genomics and diagnostics, these interventions propose new paradigms, which may confine or inverse the evolution of antibiotic resistance.
\end{abstract}

Keywords: antibiotic resistance, inversion, mutations, bacteria, evolution

\section{Foreword}

Antibiotics are the legacy of medicine; however, their competence is severely exposed to the evolution of the resistance. Since the discovery of antibiotics, the resistance has been detected and documented as an emerging threat; at present, almost all the firstgeneration antibiotics are ineffective. Basically, the main concern is the use of antibiotics as they not only stop the infections but also are associated with the evolution and emergence of the resistance, which clearly oppose the continuing use of antibiotics. ${ }^{1}$ The inappropriate use of antibiotics including self-medication and the overprescribed antibiotic therapy especially in outpatient departments for nonbacterial respiratory tract infections are the most important factors that have contributed to the menace of antibiotic resistance. ${ }^{2,3}$

Up till now, this crisis has been managed by discovering the new classes of antibiotics and continued alterations in existing compounds. ${ }^{1}$ It is generally believed that the controlled use of specific antibiotics may nullify the advantage of selection and restore the general antibiotic sensitivity with the passage of time; on the other hand, years of practice and experience have revealed that antibiotic resistance is not easy to handle. Additionally, same is the case for the antibiotics that are given with various compounds that restrict the precise resistance mechanism; this line of action helped in enhancing and expanding the antibiotics spectrum. Owing to these problems, we need to develop novel ways that may stop the evolution of antibiotic resistance, turn the resistant bacterial population into the susceptible once again, or completely invert the selection of antibiotic resistance. ${ }^{1,4}$ 


\section{Advancements}

Recently, it has been found that there are certain means that may be used to reverse the evolution of antibiotic resistance and they may also be helpful to invert the selective advantage of resistant bacterial strains. However, the single-drug therapy approach always facilitates bacteria to have the selective advantage to antibiotic resistance; combined antibiotics may stop the growth of bacteria and disfavor the resistance to the single drug. ${ }^{1,5,6}$ Techniques have been developed that influence the specific physiological and evolutionary interactions between antibiotics, which as a result directly confer a disadvantage to resistant bacteria. First, if an antibiotic partly represses the efficacy of another, resistance to the first antibiotic would remove its protection to second compounds, which pose a disadvantage to resistant strains. Second, mutations associated with antibiotic resistance may be countered if these drugs produce a synergistic effect between antibiotics and other compounds. ${ }^{5}$

Modern technical advancements have permitted comprehensive systematic endeavors to find out the drug interfaces and their basic mechanisms. There are various studies that have revealed the antagonistic, synergistic, and suppressive effects between combinations of two antibiotics. ${ }^{78}$ Moreover, interactions between compounds with slight or no antibacterial activity and antibiotics are also shown. Study of pairwise interactions among different drugs has displayed that drugs having a similar mechanism of action contain an approximately analogous pattern of interaction proposing that drug interaction works via basic cell physiology and not via chemical interactions of these compounds directly. ${ }^{9-11}$ Though, in case of a number of drugs, the exact mode of interaction of drug combinations is still undefined, modern studies have explained the phenomenon behind various interactions: sudden inhibition of various steps in the pathway produces a synergistic effect between sulfa drugs and trimethoprim. ${ }^{12}$ It has been found that the nonoptimal gene regulation steers the suppressive interactions between fluoroquinolones and tetracyclines. ${ }^{11}$ Moreover, mutations in ATP synthesis and polysaccharide reform a range of interactions among antibiotic combinations. ${ }^{13}$ The recent studies have targeted the SOS response as a promising target to develop therapeutics that may help to decrease the emergence of antibiotic resistance. In future, targeting the SOS response can develop as a potential adjuvant strategy to revive our existing antibiotic collection; however, the molecular targets and related antimicrobial agents for such therapy are not much clear. ${ }^{14}$

Finally, there may be a compromise among resistances to various antibiotics; for example, resistance against one compound creates collateral sensitivity to other compound or to an antibiotic, which has resistance-mediated toxicity. Rapid mutation or acquired genes, which confer resistance against certain drugs, may enhance or reduce the resistance to the other antibiotic. ${ }^{5}$ All these negative and positive interactions among the drugs are known as collateral sensitivity and cross-resistance, respectively. In case of resistance caused by spontaneous mutations, these interactions have been studied, which showed the presence of both collateral sensitivity and cross-resistance among various antibiotic pairs. This mechanism is not exclusive for antibiotics and bacteria; it also has been observed in HIV therapeutics, malaria, cancer therapeutics, and in various plants with resistance to different pesticides. ${ }^{5,6,15}$ Significantly, in contrast to physiological interactions, combination therapy is not required in case of cross-resistance; however, it may be an evolutionary response to an antibiotic. In recent studies, it has been observed that with some exceptions, cross-resistance is found to be positive. Aminoglycosides are associated with negative cross-resistance due to modified proton motive force linked with antibiotic resistance. ${ }^{6,15}$ These methodologies may be employed to reverse the selection advantage of resistant bacterial strains, which are competing with sensitive strains and may possibly reduce the rate of the resistance evolution, or it may lead the resistant bacteria toward the antibiotic sensitivity once again.

\section{Concerns}

Considerable obstacles persist for the application of inversion therapeutic strategies in clinical settings. Antibiotic therapy choices should be made professionally, while the diagnosis of clinical infection may span over days despite the cutting-edge technologies. Additionally, the ideal choice of the therapeutic approaches is associated with bacterial genetics and their mechanisms of antibiotic resistance. Hence, clinical application of inversion therapeutic strategies would require rapid and novel diagnostic tools, which may detect the future evolutionary potential of a bacterium toward antibiotic resistance in addition to its existing resistance. These genetic diagnostic tools may also be helpful to update the therapeutics, the level of resistance to particular antimicrobial agents and the pathways that are used by these pathogens for resistance, and the discovery of novel mechanisms of resistance. Furthermore, in vitro data on selection inversion need to be validated through in vivo studies. Various approaches, which depend on combination therapy, are even more complex by pharmacokinetics that could differ across antibiotics. In addition to that, a specific drug interaction may 
alter in various genetic and environment settings or with the time as bacteria evolve.

In conclusion, the implementation of such approaches needs a cautious ethical equilibrium between treating the patients and decreasing the antibiotic resistance in the community. Eventually, fighting antibiotic resistance would demand a range of plans, which should be able to anticipate the bacterial evolution and become accustomed to both remedy and evade resistance.

\section{Disclosure}

The authors report no conflicts of interest in this work.

\section{References}

1. Fair RJ, Tor Y. Antibiotics and bacterial resistance in the 21 st century. Perspect Medicin Chem. 2014;6:S14459-14464.

2. Smieszek T, Pouwels KB, Dolk FCK, et al. Potential for reducing inappropriate antibiotic prescribing in English primary care. J Antimicrob Chemother. 2018;73(suppl_2):ii363-ii43.

3. Ventola CL. The antibiotic resistance crisis: part 1: causes and threats. P T. 2015;40(4):277-283.

4. Lopatkin AJ, Meredith HR, Srimani JK, Pfeiffer C, Durrett R, You L. Persistence and reversal of plasmid-mediated antibiotic resistance. Nat Commun. 2017;8(1):1689.
5. Baym M, Stone LK, Kishony R. Multidrug evolutionary strategies to reverse antibiotic resistance. Science (New York, N. Y.). 2016;351(6268):aad3292.

6. Lázár V, Pal Singh G, Spohn R, et al. Bacterial evolution of antibiotic hypersensitivity. Mol Syst Biol. 2013;9:700.

7. Cokol M, Weinstein ZB, Yilancioglu K, et al. Large-scale identification and analysis of suppressive drug interactions. Chem Biol. 2014;21(4):541-551.

8. Yeh P, Tschumi AI, Kishony R. Functional classification of drugs by properties of their pairwise interactions. Nat Genet. 2006;38(4):489-494.

9. Allison KR, Brynildsen MP, Collins JJ. Metabolite-enabled eradication of bacterial persisters by aminoglycosides. Nature. 2011;473(7346): 216-220.

10. Lee HH, Molla MN, Cantor CR, Collins JJ. Bacterial charity work leads to population-wide resistance. Nature. 2010;467(7311):82-85.

11. Bollenbach T, Quan S, Chait R, Kishony R. Nonoptimal microbial response to antibiotics underlies suppressive drug interactions. Cell. 2009;139(4):707-718.

12. Nichols RJ, Sen S, Choo YJ, et al. Phenotypic landscape of a bacterial cell. Cell. 2011;144(1):143-156.

13. Chevereau G, Bollenbach T. Systematic discovery of drug interaction mechanisms. Mol Syst Biol. 2015;11(4):807.

14. Mo CY, Manning SA, Roggiani M, et al. Systematically altering bacterial SOS activity under stress reveals therapeutic strategies for potentiating antibiotics. mSphere. 2016;1(4):pii:e00163-16.

15. Imamovic L, Sommer MO. Use of collateral sensitivity networks to design drug cycling protocols that avoid resistance development. Sci Transl Med. 2013;5(204):204ra132.
Infection and Drug Resistance

\section{Publish your work in this journal}

Infection and Drug Resistance is an international, peer-reviewed openaccess journal that focuses on the optimal treatment of infection (bacterial, fungal and viral) and the development and institution of preventive strategies to minimize the development and spread of resistance. The journal is specifically concerned with the epidemiology of antibiotic

\section{Dovepress}

resistance and the mechanisms of resistance development and diffusion in both hospitals and the community. The manuscript management system is completely online and includes a very quick and fair peerreview system, which is all easy to use. Visit http://www.dovepress.com/ testimonials.php to read real quotes from published authors. 